\title{
\HIGH RESOLUTION 3D MODELING OF THE BEHAIM GLOBE
}

\author{
F. Menna ${ }^{1}$, A. Rizzi ${ }^{1}$, E. Nocerino ${ }^{1}$, F. Remondino ${ }^{1}$, A. Gruen ${ }^{2}$ \\ ${ }^{1}$ 3D Optical Metrology (3DOM) unit, Bruno Kessler Foundation (FBK), Trento, Italy \\ $<$ fmenna, rizziale, nocerino, remondino>@fbk.eu, http://3dom.fbk.eu \\ ${ }^{2}$ Institute of Conservation and Building Research, ETH Zurich, Switzerland \\ agruen@geod.baug.ethz.ch
}

Th. Session WG V/2

KEY WORDS: 3D modeling, Laser scanning, Image matching, Point cloud, Texturing, Cultural Heritage

\begin{abstract}
:
The article describes the 3D surveying and modeling of the Behaim globe, the oldest still existing and intact globe of the earth, preserved at the German National Museum of Nuremberg, Germany. The work is primarily performed using high-resolution digital images and automatic photogrammetric techniques. Triangulation-based laser scanning is also employed to fill some gaps in the derived image-based 3D geometry and perform geometric comparisons. Major problems are encountered in texture mapping. The 3D modeling project and the creation of high-resolution map-projections is performed for scientific, conservation, visualization and education purposes.
\end{abstract}

\section{INTRODUCTION}

The availability of Cultural Heritage objects in form of $3 \mathrm{D}$ digital reproductions has caused a fundamental change in our cognitive assessment and working approach. Indeed the availability of 3D digital replicas, compared to standard 2D photos, drawings or videos, generally communicates in a more effective way information about scenes or objects that have intrinsic 3D characteristics. Moreover, if metrics, accuracy and photo-realism are also taken into consideration, the $3 \mathrm{D}$ models are a powerful tool in particular for archaeological and architectural applications, not only for documentation and preservation issues, but also for study, interpretation, restoration and educational purposes. Indeed the opportunities created by $3 \mathrm{D}$ virtual environments change the way we access and exchange knowledge, leading to the recognition of new connections and enlarging the possibilities to interpret and analyze the past.

These important concepts apply to the physical model of the Earth too. In 2005 the International Cartographic Association (ICA) established the Working Group on "Digital Technologies in Cartographic Heritage" whose leading principle is that cartographic heritage is a Cultural Heritage (Hruby et al., 2006). Following this idea, the Working Group encourages to apply digital technologies to cartographic and geographic heritage for making it available and open to professionals, scientists as well as the general public. There are several advantages in using 3D virtual globes, e.g. (i) examine map contents in detail, (ii) plan and test restoration activities without risks for the original physical object and (iii) share the model with other researchers and the public.

This paper reports about the 3D surveying and modeling of the Behaim globe, the oldest still existing and intact globe of the earth, located in the German National Museum in Nuremberg, Germany. The 3D modeling project and the creation of highresolution map-projections was requested for scientific (new "reading" and analysis of the globe), conservation, visualization and education purposes. The paper reports all the steps of data acquisition and processing, analyzing critically the problems encountered and the chosen solutions for the realization of the high resolution 3D textured model of the oldest earth's globe.

\section{RELATED WORKS ON GLOBE 3D MODELING}

In the last years, several projects aimed to create digital models of both earth and celestial globes.

Most of the approaches are not much interested in producing accurate $3 \mathrm{D}$ shapes of the analyzed globes but they mainly focus on high quality textures which are projected onto a simple spherical object.

The Department of Geography and Regional Research at the University of Vienna (Austria) realized a virtual representation of the globe produced by Gerard Mercator in 1541 and now stored in the Globe Museum of the Austrian National Library (Hruby et al., 2006). This virtual representation is part of the new multimedia section of the Museum and is accessible to a wide audience via a dedicated touchscreen (Hruby et al., 2008). They employed a digital camera for acquiring 432 images which were transformed to a reference system via photogrammetric software in order to obtain an equidistant cylindrical projection. A similar method was employed by (Marton and Gercsak, 2009). They used both map prints of globes (when available) and digital photos and georeferenced them using intersections of the geographic grid as control points. Both methods resulted in a tessellation of single pictures which needed to be merged into one complete overlay. Radiometric inadequacies were corrected in post-processing in order to obtain a homogeneous appearance of the globe's overlay.

The Department of Cartography and Geoinformatics at Eötvös Loránd University (Hungary) created a Virtual Globe Museum, i.e. a collection of earth and celestial digital representations of historical globes, completely accessible through the Internet (Marton, 2012). In this project, two types of representations are used: a simple spherical object with the surface of a globe as texture (visualized as VRML file) and a KML "globe layer" which can be visualized within Google Earth.

Other approaches rely on close-range photogrammetry or triangulation-based laser scanning for the geometric and appearance modeling of the globes, trying to digitize all the small deformations and deviations from the simple spherical shape. The photogrammetric technique was applied for the first time to the Behaim globe by Kraus (Kraus and Vozikis, 1978; 
Kraus et al., 1992) in order to obtain the globe's shape and deformation. In Adami and Guerra (2008), the shape of the Coronelli's globe is digitized with a Minolta laser scanner. In Sotoodeh et al. (2008) the digitization and modeling of the St.Gallen globe was presented. The authors highlighted the main problems arising in scanning physical globes. The coregistration of the individual datasets of the sphere with the ICP algorithm, just based of geometrical surface information, can result in an ill-posed problem. Moreover, it has to be considered that the textural information is usually the most important aspect of the digitalization process.

\section{THE BEHAIM GLOBE IN NUREMBERG}

The Behaim globe, also called "Erdapfel", was built in Nuremberg around the year 1492 by a group of scholars and craftsmen supervised by Martin Behaim (Goerz and Holst, 1997). Behaim was a German navigator and geographer for King John of Portugal and created a spherical earth in opposition to the still many believers of a flat earth concept. It conforms to an idea of a globe envisioned already by Pope Sixtus IV in 1475, but has the added improvements of meridians and an equatorial line. The globe (Fig. 1) has a diameter of approximately $50 \mathrm{~cm}$ and it is now preserved in the German National Museum in Nuremberg. The famous globe contains approximately 2000 place names, 100 pictorial illustrations and more than 50 long legends. Not the quality of the information (it has some geographical inaccuracies), but its quantity and selection make the globe an important primary source for historical research. Europe, Africa and Asia are displayed on the globe surface, but not the Americas, since they were not yet discovered (Columbus arrived in the Americas on his first voyage in 1492). The globe has been restored several times, not always in a satisfying manner and now several patches are visible on the globe's surface.

The Behaim globe is a landmark not only because it is the oldest Earth model and the last tangible sample of the late medieval pre-columbian culture, but also because it is the first example of a virtual globe system. Indeed in the early 1990's a digitization work was realized for its 500 anniversary with the primary goal of providing a digital access to the globe for historians, geographers and the general public (Kager et al., 1992; Krauss et al., 1992). A photogrammetric survey was conducted in order to determine the actual form of the globe. Around 30 photographs were acquired in polarized light using a large format glass plate camera (Linhof Technica camera with a format of $84 \mathrm{~mm} \times 114 \mathrm{~mm}$ ), keeping the camera fixed and rotating the globe around its rotation axis for assuring constant lighting conditions on the globe's surface. The digitized images were triangulated with a bundle block adjustment, using ca 2000 tie points on the globe's surface and achieving a final standard deviation of $0.2 \mathrm{~mm}$. These points were then used to fit an idealized sphere (the shape of the digital model should correspond to the original globe's shape without including age- related deformation), whose radius resulted in $247.6 \mathrm{~mm}$ (3.5 $\mathrm{mm}$ std). They also produced a textured $3 \mathrm{D}$ model. Finally ortho-photographs were derived and used to create a hypermedia system which consisted of structured information units made up of texts and images (miniatures of globe details, comments, cultural and historical information) for providing comprehensive descriptions of the different globe regions (Goerz and Holst, 1997).

\section{3D SURVEYING AND MODELING}

Our data acquisition planning started from the analysis of the project requirements and location of the globe in the museum. The globe has a diameter of about $50 \mathrm{~cm}$ and is mounted in a suspension frame made of two orthogonal metallic rings that represent the celestial meridian and equator. For the survey the globe in its own stand was arranged on a trolley. The globe could rotate around its rotation axis and be moved using the trolley.

Since both the globe's geometry and high resolution color textures were required by the curators, the globe was planned to be surveyed integrating close-range photogrammetry and triangulation-based laser scanning. In this way, beside geometrical comparisons, the strength of each technique could be employed to fulfill the project requirements with minimal (manual) effort.

The globe is finely painted with colors and structures (continents, islands, text, figures, etc., see Fig. 6), thus presenting almost everywhere good texture for automated image matching algorithms. Therefore, the surveying was mainly performed using close-range photogrammetry and reconstructing the surface (shape) of the globe using dense image matching techniques. Small gaps and missing parts due to a lack of texture information (such as in areas of the oceans) were filled integrating range data acquired with a triangulationbased laser scanner.

\subsection{Image data acquisition}

The photographic session lasted one full day and played a key role in the whole survey. One of the main difficulties was the lighting condition of the museum's room characterized by different color temperatures due to a mix of direct daylight from the skylights and reflections from the surrounding blue walls. Unfortunately the globe could not be moved to another place, neither could the light be controlled by screening the windows and lamps. Therefore, a dedicated structure made of aluminum bars forming a cube was built around the globe (Fig.1d).

The structure was surrounded by white translucent drapery and three daylight photographic lamps were arranged all around the globe to produce diffuse lights. Besides a careful lighting preparation, light reflections on the sides of the images could still be observed on site due to the high curvature of the globe.
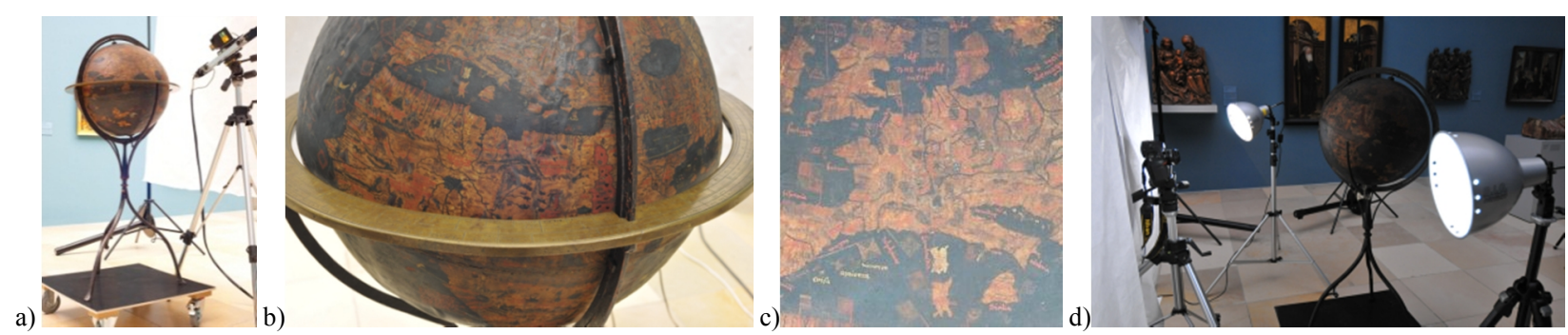

Fig. 1: The Behaim globe, preserved in the German National Museum in Nuremberg, (a) placed on a trolley during the surveying. (b) The equator and supporting ring and (c) a close view to central Europe. (d) The lighting conditions during the image acquisition. 
Even the use of circular polarizer filters on both lamps and lens had no evident effect in reducing the reflections that depend on the relative positions between camera and the lamps.

For the image acquisition the celestial equator was removed and remounted for the laser scanning acquisition session in order to assure a more robust scans alignment using an ICP algorithm.

A Nikon D3x (CMOS sensor with 24 megapixel and 6 micron pixel size), coupled with a Nikkor $50 \mathrm{~mm} \mathrm{f} / 1.4 \mathrm{D}$ lens, was employed for the acquisition of 14-bit images. In order to check the quality of the images directly on site, the camera was connected to a laptop with a calibrated screen and controlled by specific software in order to control every shot. The resolution of the texture had to be in the order of $0.1 \mathrm{~mm}$ as required by the curators, therefore the distance to the object was kept constant to ca. $600 \mathrm{~mm}$, leading to an image scale of 1:12. With these parameters the expected Ground Sample Distance (GSD) on the globe was about $0.07 \mathrm{~mm}$ at $600 \mathrm{~mm}$ from the camera and the field of view was expected to be ca $43 \times 29 \mathrm{~cm}^{2}$.

In order to assure the required resolution, some important considerations on the relation between depth of field and spatial resolution were taken into account during the image acquisition planning. Indeed, one of the most important factors to be considered in the image acquisition, when photographing in quasi macro regime, is the limited Depth of Field (DOF) that can definitely degrade the effective spatial resolution of the image. The DOF is defined as the distance between the nearest and the farthest point that is acceptable as sharp in the image and depends on the average object distance, the focal length $\mathrm{c}$ (and thus the scale number S), the F-number, and the value of the Circle of Confusion ( $\mathrm{CoC})$. The DOF can be computed as:

$$
\begin{aligned}
& \text { DOF }=\text { Farthest } \text { distance }- \text { Nearest }_{\text {distance }} \\
& =\frac{2 \cdot \operatorname{CoC} \cdot F_{\text {number }} \cdot(1+S)}{S^{2}-\left(\frac{\operatorname{CoC} \cdot F_{\text {number }}}{C}\right)^{2}}
\end{aligned}
$$

with $\mathrm{CoC}$ the diameter of the blur spot, measured on the sensor. During the globe's surveying, the blur effect caused by the defocusing had to be not larger than the resolution required. Therefore, the value of the circle of confusion was calculated as the ratio GSD/S that is equal to $8.3 \mu \mathrm{m}$ on the sensor.

Using Eq.(1), the DOF can be calculated for different values of the F-number. Thus the DOF represents a part of the object space where the size of the $\mathrm{CoC}$ is below the GSD and does not degrade the resolution required by the curator.
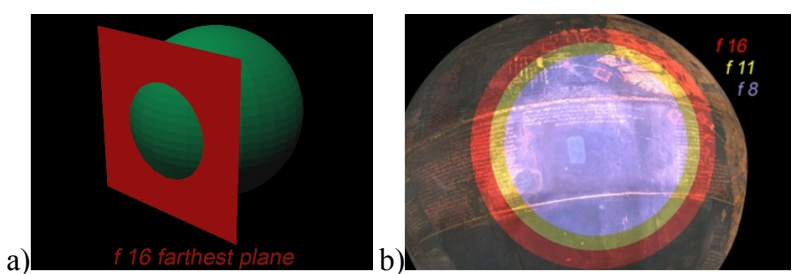

Fig. 2: (a) Spherical cap of the globe delineating the in-focus imaging area. (b) Different spherical areas according to different F-numbers.

Figure 2a shows the sphere of the globe cut by the plane located at the farthest limit distance for DOF using F-number equal to 16. The plane of nearest limit is considered to be tangent to the globe and is not depicted in the figure. The area of the image that can be effectively used for texturing and mapping is smaller than the one expected from the field of view. The parts of the globe, contained in the sharp DOF zone for F-numbers respectively equal to $8,11,16$, are spherical caps whose sizes depend on the F-number (Fig.2b). Thus, as a general rule, chosen the scale number and the focal lens, the higher the Fnumber the higher the DOF. For high F-numbers, the resolving power becomes uniform over the whole image (for most of the commercial lenses F-number is higher than 8) but at the same time, when the F-number approaches the maximum value allowed by the employed lens, the resolving power of the lens rapidly decreases due to diffraction effects.

As also reported in different resolution tests for the most popular lenses (i.e. www.dpreview.com), the Nikkor $50 \mathrm{~mm}$ $\mathrm{f} / 1.4 \mathrm{D}$ (used for the globe's survey) at F-number equal to 11 has a good compromise between resolution and depth of field and thus it was chosen for the image acquisition. At F-number equal to 11 the usable spherical cap (Fig.2b) has a boundary circle with a diameter of ca $23 \mathrm{~cm}$ which corresponds to an angle at the center of the sphere of about 55 degrees.
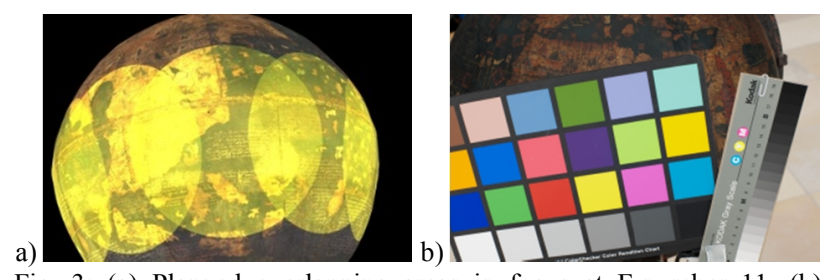

Fig. 3: (a) Planned overlapping areas in focus at F-number 11. (b) Reference color checker used for color correction and balancing.

The image acquisition was performed keeping the camera fixed on the tripod and rotating the globe by approximately 30 degrees for each photo. This angle assured a good intersection among optical rays during image triangulation by bundle block adjustment and a redundancy of images for the texturing in the most critical areas due to light reflections (Fig.3a).

The entire globe was covered with six stripes of images at different latitudes (three for each hemisphere) for a total of ca. 80 images (Fig.4a). In order to assure a high fidelity color reproduction, images of a color checker were also acquired. An additional set of 8 images was taken to scale the photogrammetric project using a $600 \mathrm{~mm}$ calibrated steel bar.

\subsection{Image data processing}

The camera interior and additional parameters were determined on site using a self-calibration approach with a portable testfield whose size was comparable to the globe.

The globe images were triangulated and oriented using an automatic tie point extraction approach (Pierrot-Deseilligny et al., 2011) and a successive photogrammetric bundle adjustment (Fig.4b). The statistical information from the bundle adjustment reports:

- the estimated standard deviation of image coordinate observations is less than 0.5 pixels;

- the average number of intersecting rays per $3 \mathrm{D}$ point is 5 (minimum is 3 );

- the average intersecting angle between homologous rays is 75 degrees (minimum is 20 degrees);

- the theoretical precision of the computed $3 \mathrm{D}$ coordinates is $0.06 \mathrm{~mm}$ (average of the three components).

After image orientation, the set of images was processed with a multi-image matching algorithm (Furukawa and Ponce, 2009) in order to derive a dense point cloud of the globe's surface (Fig.4 c, d, e, f). The stripes were processed singularly, then manually filtered and edited in order to remove some outliers and finally merged together giving a unique point cloud containing ca 10 million points with $0.3 \mathrm{~mm}$ linear resolution (sampling step). 

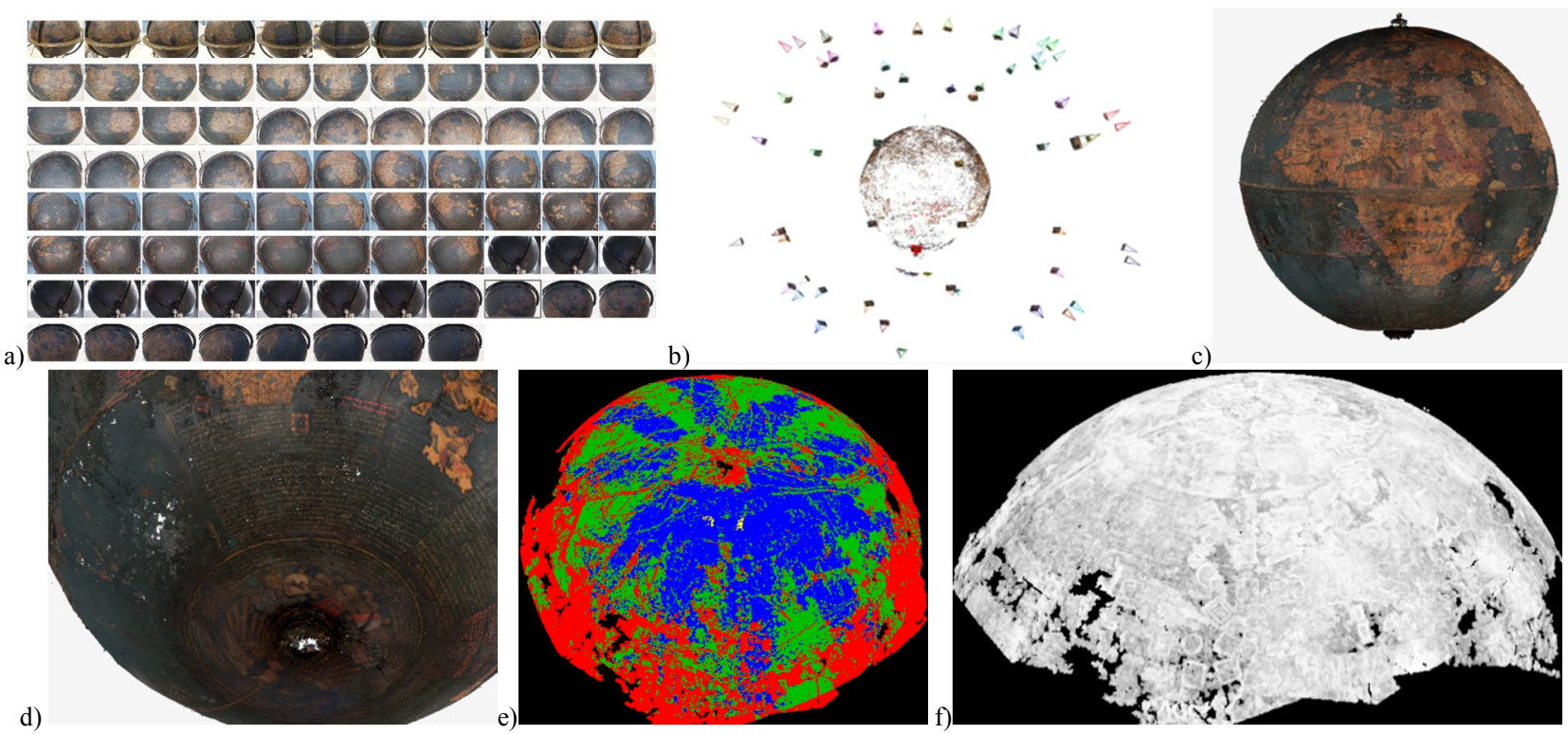

Fig. 4: (a) The set of acquired images used for the surveying and 3D reconstruction of the globe. (b) Camera poses and sparse 3D tie points after the bundle adjustment. (c) Dense cloud (10 million points) derived by image matching and (d) a closer view of the cloud with some small holes due to lack of texture or uniform areas. (e) Part of the derived point cloud in the upper area of the globe classified according to the number of rays for each $3 \mathrm{D}$ point (red=2, green $=3$, blue $=4$, yellow $=5$, cyan=6, magenta $=7$ ). (f) Part of the cloud in the upper part of the globe visualized according to the average correlation coefficient (white is higher than 0.9 , dark gray is lower than 0.4 , black is no matching).

\subsection{Range data acquisition and processing}

A Shape Grabber triangulation-based laser scanner was used for the surveying of the globe and its equators. 25 scans at $0.3 \mathrm{~mm}$ geometric resolution were acquired (ca 33 mil. points), aligned and edited producing a unique point cloud of ca 15 mil. points (Fig.5). These data are used to integrate and fill the gaps of the photogrammetric point cloud.

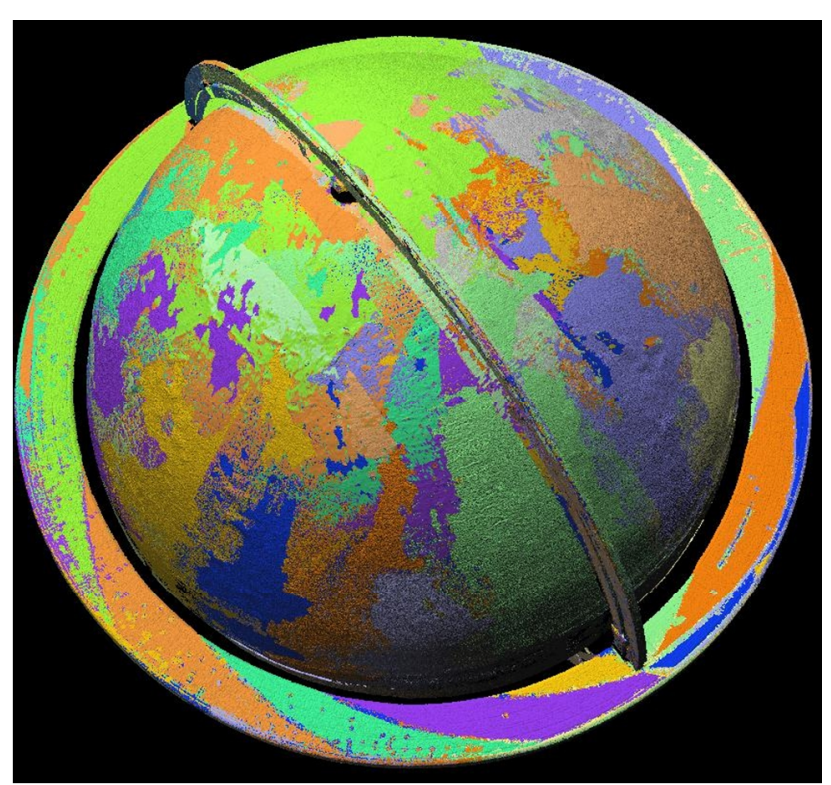

Fig. 5: Registered range-based point clouds of the Behaim globe.

\subsection{Final textured polygonal model}

The small gaps and holes in the photogrammetric point cloud were filled using the range-based point cloud. Then a triangular mesh was wrapped over the unstructured point cloud in order to create a photo-realistic 3D polygonal model of the globe.

A new texture mapping tool was developed in order to map the RGB images onto the globe's surface geometry, correct and balance the color of the images and finally create a seamless textured 3D model.

\subsubsection{RGB automatic texture mapping}

Firstly image masks were defined in order to map only in focus imaging areas. The masks were created automatically, one for each strip, by reprojecting on the images the spherical caps obtained intersecting the globe with the DOF farthest plane (sect. 4.1). Then the texture mapping algorithm, starting from the known exterior and interior orientation parameters, found the best image (or part of it) for each triangle of the polygonal model. The image selection is performed weighting different parameters like (i) the image viewing angle, (ii) the distance camera-object and (iii) the depth of field.

\subsubsection{Radiometric manual adjustment}

When multiple (at least 2) images are overlapping, each conjugate pixel should preferably have the same intensity in every image, but in reality it is not the case. The reasons are multiple, namely ambient light changes, change in the lens aperture or camera exposure time, possible image alignment errors, un-modeled lens distortion, decrease in intensity towards image edges ("vignetting"), etc. Thus, in order to create a seamless texture mapping result, a good blending strategy must be applied (Uyttendaele et al., 2001; Brown and Lowe, 2003; Agarwala et al., 2004; McCann and Pollard, 2008).

The circular patches used to texture the globe's geometry overlap mainly at their borders. These areas needed to be radiometrically equalized as well as other areas (even in the center of the patches) due to specular reflections. As blending function, the method proposed in (Porter and Duff, 1984) was implemented using a MaxScript. Given a template (tem) and a reference $(r e f)$ images, the color and alpha components $c$ and $a$ of the final radiometrically correct image ( fin) are given by:

$$
\begin{gathered}
C_{f i n}=f\left(C_{r e f}, C_{t e m}\right) a_{t e m} a_{r e f}+Y C_{t e m}\left(1-a_{r e f}\right) \\
+Z C_{r e f}\left(1-a_{t e m}\right) \\
a_{f i n}=X a_{r e f} a_{t e m}+Y a_{r e f}\left(1-a_{t e m}\right)+Z a_{t e m}\left(1-a_{r e f}\right)
\end{gathered}
$$


International Archives of the Photogrammetry, Remote Sensing and Spatial Information Sciences, Volume XXXIX-B5, 2012 XXII ISPRS Congress, 25 August - 01 September 2012, Melbourne, Australia
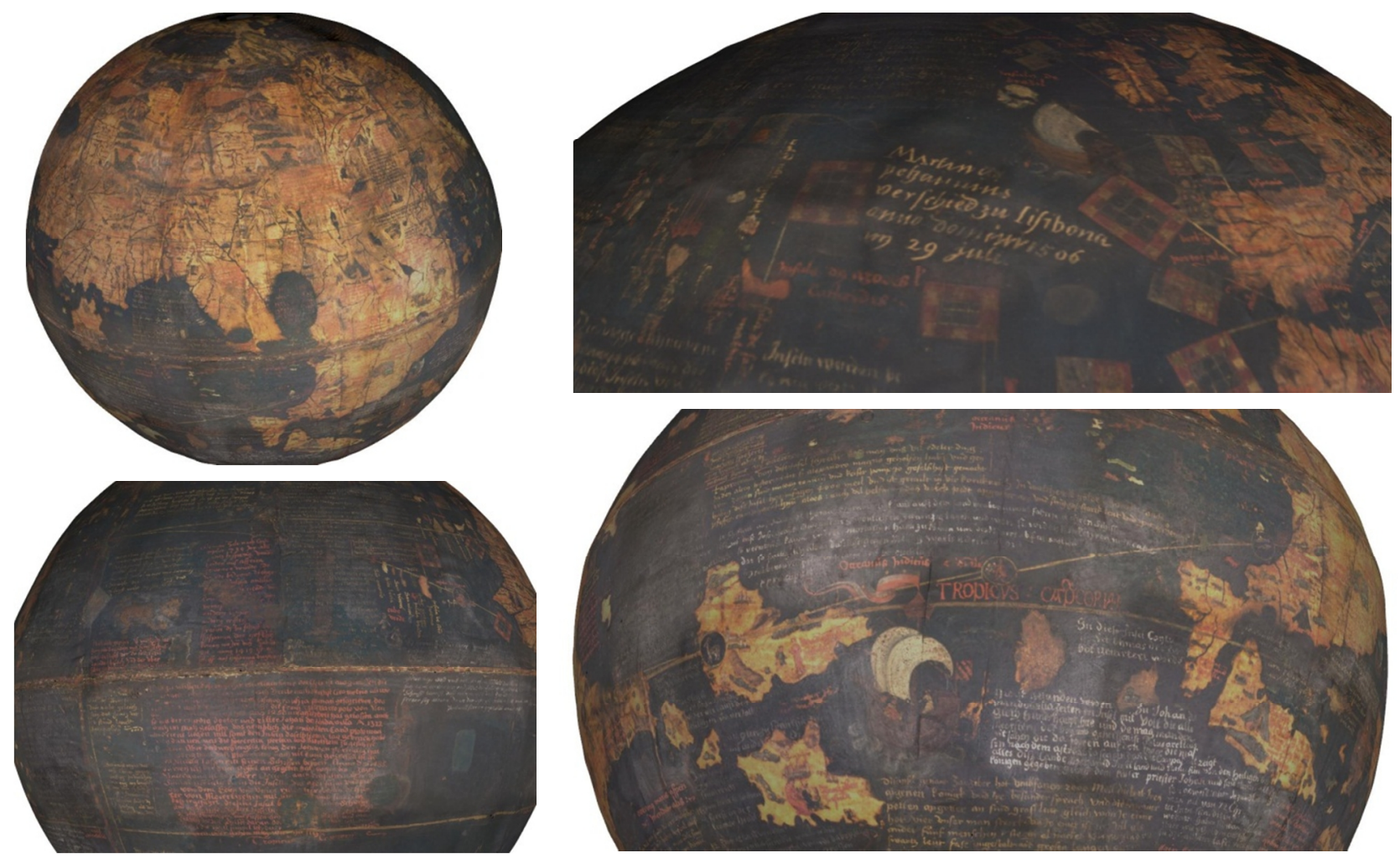

Fig. 6: The textured model of the globe with some closer views of the illustrations and legends.

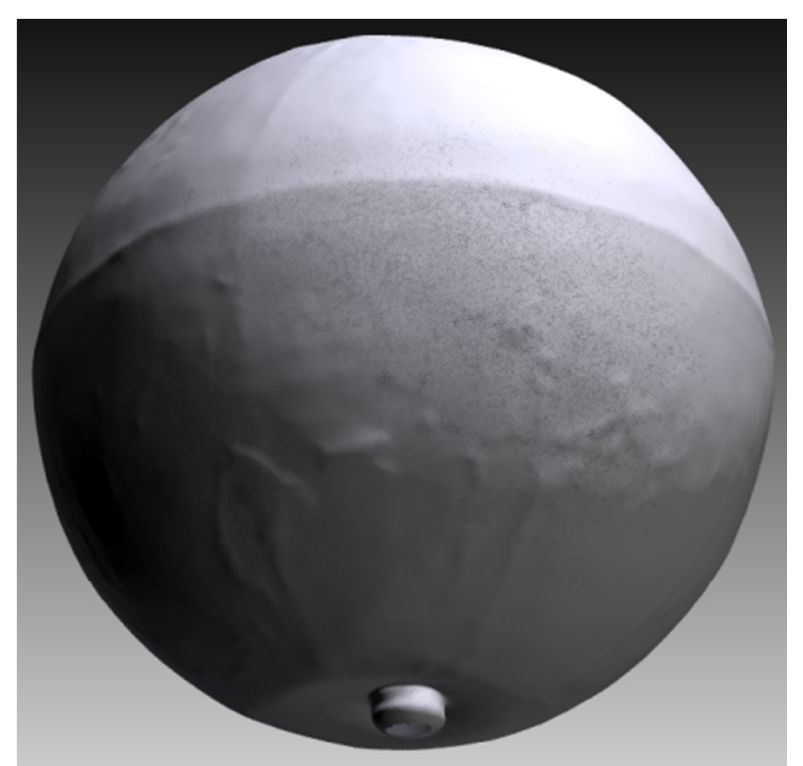

Fig. 7: A 1 million polygons decimated mesh of the globe. Small geometric imperfections ("bubbles") are still visible despite the lowresolution geometric model.

where $f\left(c_{\text {ref }}, c_{\text {tem }}\right)$ returns a value based on the reference and template color components. The parameters $\mathrm{X}, \mathrm{Y}$ and $\mathrm{Z}$ are either 0 or 1 and their permutations produce various blending modes.

The final textured 3D model (Fig.6) counts more than 24 million polygons, while the texture requires more than 5 Gigabytes. Therefore, for interactive visualization and for webapplications, reduced versions (in geometry and texture) were also created (Fig.7).

\section{ANALYSES AND PROJECTIONS}

A best-fitting sphere was then computed using the reconstructed point cloud (ca 10 million points). The estimated radius of the ideal sphere resulted in $247.4 \mathrm{~mm}$ with a standard deviation of $3.1 \mathrm{~mm}$. Error maps between the ideal sphere and the reconstructed one show $2 \mathrm{~cm}$ differences at the poles and approximately $8 \mathrm{~mm}$ along the equator (Fig. 8).

A reference system was then defined with its center in the center of the ideal sphere, the XY plane derived fitting a plane through some manually measured points along the globe's equator. Some other points measured along a meridian helped to derive another plane whose intersection with the equator plane gave the direction of the $\mathrm{X}$-axis.

According to the defined reference system, map projections were also realized, representing the globe's surface on a plane. The equirectangular projection was selected (Fig.9). It is a cylindrical projection which neither stretch nor compress northsouth distances thus preserving distances along the meridians and the equator. It maps meridians to vertical straight lines of constant spacing and circles of latitude to horizontal straight lines of constant spacing. The projection is neither equal area, equal distance nor conformal.

\section{CONCLUSIONS AND LESSONS LEARNED}

The paper reported the 3D surveying and modeling of the oldest and still existing globe of the earth, the Behaim globe. Photogrammetry and automated matching techniques were used to derive a dense point cloud of the globe's surface and shape. Some holes of the photogrammetric cloud were closed using geometric data acquired with a triangulation-based laser scanner. A least squares adjusted sphere was computed for comparison with the actual globe shape while map projections were realized for documentation and analyses purposes. 
International Archives of the Photogrammetry, Remote Sensing and Spatial Information Sciences, Volume XXXIX-B5, 2012 XXII ISPRS Congress, 25 August - 01 September 2012, Melbourne, Australia

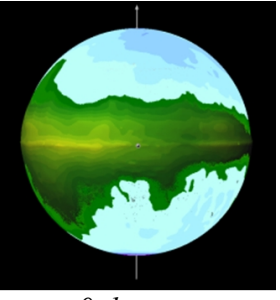

0 degrees

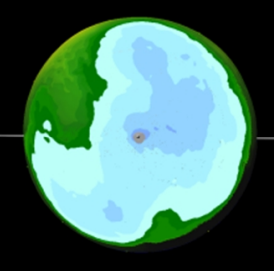

North pole

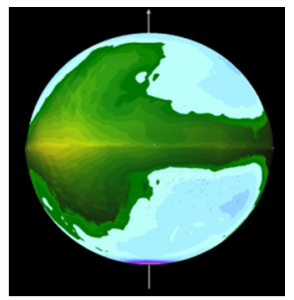

180 degrees

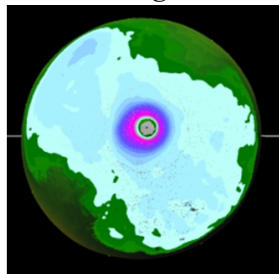

South pole

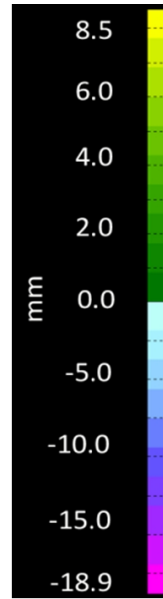

18.9
Fig. 8: Error maps between the best-fitted sphere and the globe's shape.

Despite the highest level of attention employed during the data acquisition, radiometric differences between the images caused different problems. Firstly in the automated image matching, but the main difficulties emerged during the texturing of the geometric model (Table 1). A long manual radiometric editing was thus necessary in order to achieve a homogeneous appearance of the globe's texture which has a final resolution of $0.1 \mathrm{~mm}$. According to our experience gained in many other reality-based modeling projects, the ratio between data acquisition and processing is normally 1:10.

\begin{tabular}{|c|c|c|}
\hline Data acquisition & Geometric modeling & Appearance modeling \\
\hline 1 & 7 & 15 \\
\hline
\end{tabular}

Table 1: Approximate time ratio between the data acquisition, and the realization of the final textured 3D model of the globe.

The project is still on-going and the next steps include the creation of LOD-based versions of the 3D globe for different purposes (web-based visualization, sharing, restoration, analyses, etc.) and the comparison between the employed images with older ones (to highlight changes and texture degradations).

\section{REFERENCES}

Adami A., Guerra F, 2008: Coronelli's Virtual Globe. e-Perimetron, Vol. 3(4).

Agarwala, A., Dontcheva, M., Agarwala, M., Drucker, S., Colburn, A., Curless, B., Salesin, D., Cohen, M., 2004: Interactive digital photomontage. ACM Transactions on Graphics (SIGGRAPH’04).
Furukawa, Y., Ponce, J., 2010: Accurate, dense, and robust multi-view stereopsis. IEEE Trans. on PAMI, Vol.32(8): 1362-1376.

Goerz, G., Holst N., 1997: The digital Behaim globe (1492). Proc. $4^{\text {th }}$ Inter. Conference on Hypermedia and Interactivity in MuseumsICHIM, pp. 157-173.

Hruby, F., Plank, I., Riedl, A., 2006: Cartographic heritage as shared experience in virtual space: a digital representation of the earth globe of Gerard Mercator (1541). e-Perimetron, Vol.1(2): 88-98.

Hruby, F., Riedl, A., Tomberger, H., 2008: Virtual representations of antique globes - new ways of touching the untouchable. Inter. Journal of Digital Earth, Vol.1(1): 107-118.

Kraus, K., Vozikis, E., 1978. Die Verebnung von Globen mit Hilfe der Differentialumbildung. Globe studies, pp. 55-61.

Kager, H., Kraus, K., Steinnocher, K., 1992: Photogrammetrie und digitale Bildverarbeitung angewandt auf den Behaim-Globus. Zeitschrift für Photogrammetrie und Fernerkundung, Vol.5/92:142-148.

Kraus, K., Tschannerl, J., Halmer, A., Rottensteiner, F., 1992: Die Bearbeitung des Behaim-Globus mit photogrammetrischen Methoden. Globe studies, 40/41: 9-19.

International Cartographic Association (ICA) - Working Group on "Digital Technologies in Cartographic Heritage", http://xeee.web.auth.gr/ICA-Heritage/2011 2015/History.htm (accessed April 15th, 2012)

Marton M., Gercsak G., 2009: Virtual globes museum a tool for safeguarding, communicating and teaching cultural heritage. Proc. 24th International Cartographic Conference, Chile.

Marton 2012: Virtual Globes Museum, http://terkeptar.elte.hu/vgm/?lang=en (accessed April 15th, 2012).

McCann, J., Pollard, N.S., 2008: Real-time gradient-domain painting. ACM Transaction of Graphics (SIGGPRAPH'08), Vol.27(3).

Pierrot-Deseilligny, M., De Luca, L. and Remondino, F., 2011. Automated image-based procedures for accurate artifacts 3D modeling and orthoimage generation. 23th Int. CIPA Symposium, Prague, Czech Republic (on CD-ROM).

Porter, T., Duff, T., 1984: Compositing digital images. Computer Graphics, Vol. 18(3): 253-259.

Sotoodeh, S., Gruen, A., Hanusch, T., 2008: Integration of structured light and digital camera image data for the $3 \mathrm{D}$ reconstruction of an ancient globe. Inter. Archives of the Photogrammetry, Remote Sensing and Spatial Information Sciences. Vol. 37(B5), Beijing, China.

Uyttendaele, M., Eden, A., Szeliski, R., 2001: Eliminating ghosting and exposure artifacts in image mosaics. Proc. CVPR01, Vol. 2: 509-516.
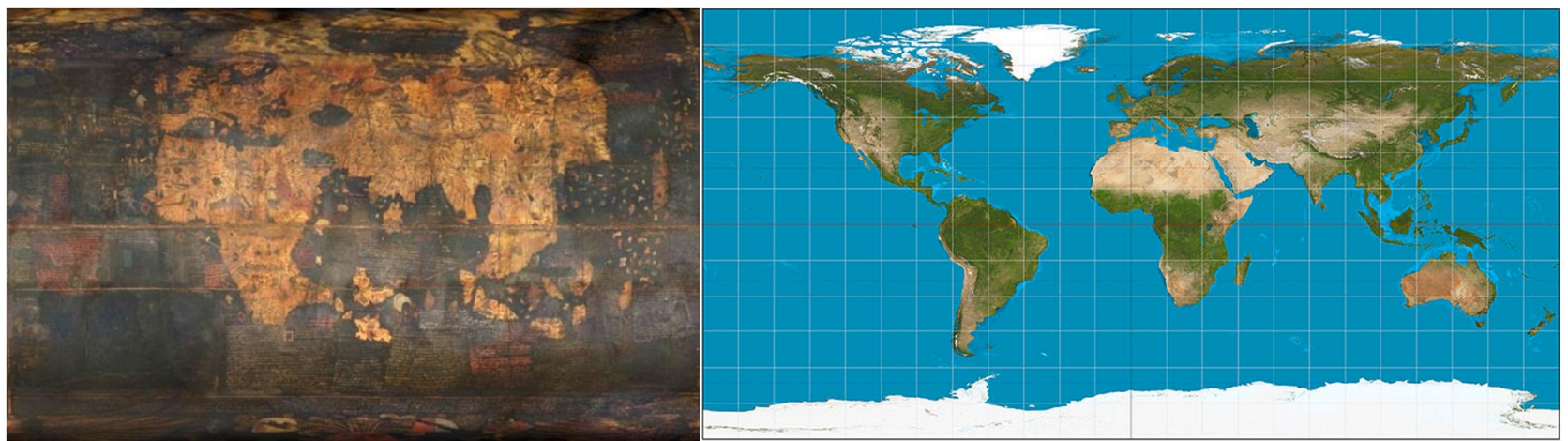

Fig. 9: Equirectangular projection of the Behaim globe compared to the actual world. 